\title{
Correlation-Induced Electron-Electron Attraction
}

\author{
Hubert Klar \\ University of Freiburg, Freiburg, Germany (Retired) \\ Email: HubKlar@aol.com
}

How to cite this paper: Klar, H. (2018) Correlation-Induced Electron-Electron Attraction. Journal of Modern Physics, 9, 1761-1772.

https://doi.org/10.4236/jmp.2018.99110

Received: June 17, 2018

Accepted: July 30, 2018

Published: August 2, 2018

Copyright (C) 2018 by author and Scientific Research Publishing Inc. This work is licensed under the Creative Commons Attribution International License (CC BY 4.0).

http://creativecommons.org/licenses/by/4.0/

\begin{abstract}
The multidimensional potential surface of a few (2 - 4)-electron atom shows near equilibrium configurations ridge structures. The electron wave diffraction from such a ridge is shown to induce a novel fictitious force which manifests itself as temporary electron-electron attraction. In contrast to a Cooper pair our pair does not need a lattice vibration and may be regarded as elementary prototype pair in an isolated atom. Also electron triples and quadruples are discussed.
\end{abstract}

\section{Keywords}

Cooper Pairs, Dominant Correlation, Wannier Theory, Multiple Excitation

\section{Introduction}

Electronic structure of atoms and molecules is often well understood within the shell model. Each electron moves in a potential field generated by all other electrons. Principal quantum numbers and angular momentum quantum numbers describe approximately the state of individual electrons.

That traditional picture, however, breaks down at high multiple excitation, particularly near thresholds of multiple escape. The reason for that breakdown is that the electrons move no longer in potential valleys. This may be seen in the following simple model.

To this end, we consider a two-electron-atom in a collinear configuration, the nucleus located between the electrons. The electron-electron distance may be

$$
\rho=r_{1}+r_{2}
$$

the $r_{i}, i=1,2$, being the electron-nucleus separations. The condition (1) may be satisfied by the parametrization 


$$
\begin{aligned}
& r_{1}=\rho \varepsilon \\
& r_{2}=\rho(1-\varepsilon)
\end{aligned}
$$

with $0<\varepsilon<1$. The electrostatic potential of this atom with nuclear charge $Z$

$$
V=-\frac{Z}{r_{1}}-\frac{Z}{r_{2}}+\frac{1}{\rho}
$$

reads then as function of the parameters $\varepsilon$ and $\rho$

$$
V=\frac{1}{\rho}\left(-\frac{Z}{\varepsilon}-\frac{Z}{1-\varepsilon}+1\right)
$$

As long as $\varepsilon \approx 0$ or $\varepsilon \approx 1$ one electron is close the nucleus and experiences a Coulomb field. The electrons experience however no hydrogen-like force at all if

$$
\frac{\mathrm{d} V}{\mathrm{~d} \varepsilon}=0
$$

which has the solution $\varepsilon=1 / 2$. At that $\varepsilon$-value the electrons have equal distances from the nucleus and are trapped in an equilibrium configuration. The Taylor expansion of (4) at $\varepsilon=1 / 2$ reads

$$
V \approx-\frac{4 Z-1}{\rho}-\frac{16 Z}{\rho}\left(\varepsilon-\frac{1}{2}\right)^{2}
$$

Equation (6) identifies this equilibrium as unstable because $\frac{\partial^{2} V}{\partial \varepsilon^{2}}<0$. Along the coordinate $\varepsilon$ the potential shows an antioscillator, in the $(\rho, \varepsilon)$-scattering plane (6) represents a ridge. The importance of potential ridges for electronic excitation has been stressed recently [1].

The above model of a synthetic atom is not entirely unrealistic. Wannier [2] has studied the threshold ionization of $\mathrm{H}$ by electron impact. His model has been as follows. He assumes that the projectile electron transfers its kinetic energy onto the target electron. We are then confronted with two extremely slow electrons in the field of the nucleus. We meet here exactly the situation described above; the projectile electron moves near a potential ridge. Within the framework of classical Lagrange equations Wannier extracted from the decay of this negative complex the slope of the total ionization cross section. He found a power law with fractional exponent,

$$
\sigma \propto\left(E-E_{0}\right)^{1.127 \cdots}
$$

where the fractional exponent stems from the curvature of the potential surface along the unstable equilibrium.

The present paper goes fare beyond the classical Wannier work, and also beyond synthetic models. We correctly employ quantum theory to a real atom, and intend to investigate the electrons motion near unstable equilibria.

The paper is organized as follows. In Section 2, we discuss the choice of suitable coordinates for two active electrons. Coordinates are suitable if the ridge structure of the potential can easily be described, and if the expression of the ki- 
netic energy is not too difficult. We have here to fight against two mathematical problems. 1) In consequence of correlation three/more-body Coulomb problems are not separable in any coordinate system. 2) Even if correlation is properly taken into account by suitable coordinates we arrive at non-adiabatic couplings [3] [4]. The present paper presents a coordinate frame which treats properly correlation near thresholds for multiple ionization, and eliminates at least in first-order non-adiabatic coupling. This repairs a fundamental short-coming of the familiar Born-Oppenheimer method.

The method mentioned above has been recently outlined in a broader context [1]. Section 3 presents a detailed description in the example of two active electrons. Section 4 compares our quantum treatment with the corresponding classical one. It is usually believed that Wannier's cross section Formula (7) is an exact result although Wannier's paper has nothing to do with quantum theory. Section 5 finally shows that the phenomenon of wave diffraction from a ridge is a classical as well as a quantum effect. Section 6 discusses the formation of few-electron complexes like pairs and triples. Section 7 stresses breathing motions of electron charge clouds in contrast to Bohr orbits in an atom.

\section{Alternative Coordinates for 2-Electron Atoms}

Single electron coordinates are certainly not suitable in the spectral range of high multiple excitation where correlation is dominant. We start with the trivial remark that a three-body system (nucleus +2 electrons) needs in the centre-of mass system 6 space coordinates. We do only a small mistake if we put the centre of mass into the nucleus. Three of the six coordinates may be used to describe the orientation of the whole atom in space. Euler angles serve for that. We put therefore

$$
\boldsymbol{r}_{i}=D(\alpha, \beta, \gamma) \boldsymbol{b}_{i}
$$

$i=1,2$. Here the vectors $\boldsymbol{r}_{i}$ are space-fixed electron positions, the $\boldsymbol{b}_{i}$ are the body-fixed coordinates, and the $D(\ldots)$ is an orthogonal rotation matrix depending on Euler angles. For S-states treated below we have $\boldsymbol{r}_{i}=\boldsymbol{b}_{i}$.

Hyperspherical coordinates are most convenient in situations where correlation is important or even dominant. One coordinate is the hyperradius given by

$$
R=\sqrt{r_{1}^{2}+r_{2}^{2}}
$$

where $r_{i}(i=1,2)$ are the electron nucleus separations.. Two body-fixed hyperspherical angles may be expressed in terms of moments of inertia. We denote the principal axes by X, Y ynd Z such that X and Y ly in the particles plane. Previous papers by the author [3] have employed the inertia asymmetry given by

$$
\psi=\frac{1}{2} \cos ^{-1} \frac{I_{y y}-I_{x x}}{I_{z z}}
$$

as one angle where the principal axes system has been oriented according to $I_{x x} \leq I \leq I_{z z}$ for the principal moments. The value $\psi=0$ describes then all col- 
linear particle configurations. Radial correlation we describe following Sommerfeld [5] by the angle

$$
\varphi=\tan ^{-1} \frac{r_{1}^{2}-r_{2}^{2}}{4 r_{1} \cdot r_{2}}
$$

which basically is the difference of single electron moments of inertia.

The key point is now that the inertia sphere is spanned by the vector

$$
\boldsymbol{c}=R\left(\begin{array}{c}
\cos 2 \psi \cos \varphi \\
\cos 2 \psi \sin \varphi \\
\sin 2 \psi
\end{array}\right)
$$

i.e. the angle $\psi \quad(0 \leq \psi \leq \pi / 4)$ is a latitude spherical angle ${ }^{1}$ and $\varphi$ $(0 \leq \varphi \leq 2 \pi)$ is the corresponding azimuth. In consequence of that, $\varphi$ is a cyclic coordinate in the kinetic energy which makes the integration of the wave equation relatively simple. Alternative hyperspherical angles like

$$
\alpha=\tan ^{-1} \frac{r_{1}}{r_{2}}
$$

do not show that convenient property. In terms of our inertia coordinates the study of radial correlation in the collinear configuration reduces exactly to a 2-dimensional problem in the equatorial plane of the inertia sphere.

The electrostatic potential of the system electron-nucleus-electron

$$
V=-\frac{Z}{r_{1}}-\frac{Z}{r_{2}}+\frac{1}{r_{12}}
$$

where $r_{12}$ is the electron-electron separation and $Z$ being the nuclear charge shows a saddle point located at $\psi=0$ and $\varphi=\pi$. The potential (13) has in that saddle point the following Taylor expansion

$$
V=\frac{1}{R}\left[-C_{0}+\frac{C_{1}}{2} \psi^{2}-\frac{C_{2}}{2}(\varphi-\pi)^{2}\right]
$$

the coefficients given by

$$
\begin{aligned}
& C_{0}=\frac{4 Z-1}{\sqrt{2}} \\
& C_{1}=\frac{1}{\sqrt{2}} \\
& C_{2}=\frac{12 Z-1}{4 \sqrt{2}}
\end{aligned}
$$

We fall to the collinear configuration by fixing $\psi=0$.

The expansion (14) has been obtained as follows. We express the potential (13) in terms of the coordinates given in (18) and perform a Taylor expansion in the two variables $\varphi$ and $\psi$.

The Laplacian reads in our coordinates

$$
\Delta=R^{-5} \frac{\partial}{\partial R} R^{5} \frac{\partial}{\partial R}-\frac{\Lambda^{2}}{R^{2}}
$$

${ }^{1}$ Mathematians usually count the latitude by the angle $\vartheta=\pi / 2-2 \psi$. 
with the grand angular momentum in the collinear configuration given by

$$
\Lambda^{2}=4 \frac{\partial^{2}}{\partial \varphi^{2}}
$$

Equation (17) confirms that $\varphi$ is a cyclic coordinate.

These coordinates $(R, \psi, \varphi)$ are fare from new. They were already used long ago by Sommerfeld [5], see also [6]. The single electron positions read in these body-fixed inertia coordinates

$$
\begin{aligned}
& \boldsymbol{b}_{1}=R\left(\begin{array}{c}
\cos \psi \cos \frac{1}{2}\left(\varphi+\frac{3 \pi}{2}\right) \\
\sin \psi \sin \frac{1}{2}\left(\kappa+\frac{3 \pi}{2}\right) \\
0
\end{array}\right) \\
& \boldsymbol{b}_{2}=R\left(\begin{array}{c}
\cos \psi \cos \frac{1}{2}\left(\varphi-\frac{3 \pi}{2}\right) \\
\sin \psi \sin \frac{1}{2}\left(\varphi-\frac{3 \pi}{2}\right) \\
0
\end{array}\right)
\end{aligned}
$$

We are now arrived probably at the most convenient way to study an electron motion near a potential ridge. The ridge occurs in the $(R, \varphi)$ plane. The curvature across the ridge is $-C_{2} / 2 R$. The potential on the top of the ridge reads $-C_{0} / R$. In the ridge region we arrive at the following reduced stationary wave equation.

$$
\left[-\frac{1}{2} \frac{\partial^{2}}{\partial R^{2}}-\frac{4}{2 R^{2}} \frac{\partial^{2}}{\partial \varphi^{2}}+\frac{15}{8 R^{2}}-\frac{C_{0}}{R}-\frac{C_{2}}{2 R}(\varphi-\pi)^{2}-E\right]\left(R^{-\frac{5}{2}} \Psi\right)=0
$$

where the centrifugal term emerges from the elimination of the first derivative $\frac{\partial}{\partial R}$.

This is a convenient way to start the diffraction study of an electron from a potential ridge. (19) describes a two-dimensional wave propagation in the $(R, \varphi)$ plane. Geometrically we are confronted with the diffraction of an electron from a potential ridge. Correlation has been properly taken into account by the use of the above inertia coordinates. But we have paid a high price for that achievement since (19) is not separable.

\section{Non-Adiabatic Solution of the Wave Equation (19)}

The wave equation for two-electron atoms in hyperspherical coordinates has been investigated using an adiabatic approach by Macek [3] and by Klar et al. [4], i.e. we treat at first the angular motion at fixed values of $\mathrm{R}$. That step generates a potential which controls the radial motion. Macek [3] has used these angles $(\alpha, \Theta)$ for that, Klar et al. [4] did it using the convenient angles $(\psi \cdot \varphi)$. 
Although the latter method has led to some computational advantages, but both methods failed at high double excitation where correlation becomes dominant.

Nevertheless, these methods are in principle exact provided we could go beyond the adiabatic approximation. That would however require the treatment of an infinity of coupled differential equations. A truncated set would not solve the problem.

For the less experienced reader we demonstrate how an adiabatic solution of (19) looks like. We determine at first a socalled channel function, i.e. the angular eigenfunction at constant values of $R$. The channel equation

$$
-\frac{2}{R^{2}} \frac{\partial^{2} \Phi}{\partial \varphi^{2}}-\frac{C_{2}}{2 R}(\varphi-\pi)^{2} \Phi=U \Phi
$$

has the eigenfunction

$$
\Phi_{\text {adiabatic }}=\exp \left(i \kappa(\varphi-\pi)^{2}\right)
$$

to which belongs the wave number

$$
\kappa_{\text {adiabatic }}=\frac{1}{4} \sqrt{C_{2} R}
$$

and the eigenvalue

$$
U_{\text {adiabatic }}-i R^{-3 / 2} \sqrt{C_{2}}
$$

It is evident that (20) is not Hermitian, and has therefore no real eigenvalues. Equation (22) indicates where the difficulty is located. We observe that the wave number $\kappa$ depends explicitely on the coordinate $R$. Therefore the derivative of the channel function $\frac{\partial \Phi}{\partial R}$ must be taken into account in the radial wave equation.

A recent paper [1] has shown how to go beyond the above shortly outlined adiabatic approach. We still rely on a product form of the wave function,

$$
\Psi(r, \varphi)=R^{5 / 2} F(R) \Phi(\varphi ; R)
$$

Because all potential terms, see (14), scale smoothly with $1 / R$, and also because Wannier's threshold ionization law is a classical result, we employ for the radial function an Eikonal Ansatz. An improved channel function may still have the structure (21) except thar the wavenumber must be properly determined. It was pointed out by Wannier [2] that near threshold $E=0$ we have to distinguish two different regions of the hyperradius $R$. The Coulomb zone extends from the core radius $R_{0}$ to infinity and the reaction zone with $0 \leq R \leq R_{0}$ where the core is limited by the centrifugal barrier, see (19).

To this end we try to find an improved solution of (19) with help of the Ansatz

$$
R^{-5 / 2} \Psi=\exp \left(i \int^{R} K\left(R^{\prime}\right) \mathrm{d} R^{\prime}\right) \times \exp \left(i \kappa(\varphi-\pi)^{2}\right)
$$

We take powers of $\varphi$ up to the quadratic term $(\varphi-\pi)^{2}$ into account but dis- 
regard higher order power terms. To this end we arrive at two conditions for the wave numbers

$$
\begin{gathered}
K^{2}=2\left(E-\frac{15}{8 R^{2}}+\frac{C_{0}}{R}+\frac{i}{2} \frac{\mathrm{d} K}{\mathrm{~d} R}+\frac{4 i \kappa}{R^{2}}\right) \\
\frac{8}{R^{2}} \kappa^{2}=\frac{C_{2}}{2 R}-K \frac{\mathrm{d} \kappa}{\mathrm{d} R}+\frac{i}{2} \frac{\mathrm{d}^{2} \kappa}{\mathrm{d} R^{2}}
\end{gathered}
$$

Equations (26) and (27) constitute a set of two coupled Riccati equations. This is an exact result within our frame of taking into account terms up to $\varphi^{2}$ in the wave equation. We stress that we have taken into account the first and the second derivative with respect to $R$ of the angular function $\exp \left(i \kappa(\varphi-\pi)^{2}\right)$.

Near threshold ( $E \cong 0$ ) we can do a big simplification. Since all potential terms scale as $1 / R$ we are close to the classical limit of quantum mechanics. For that reason we had already employed an Eikonal approach in (25). Remember that the Eikonal approximation is exact for Coulomb potentials. Within a WKB spirit we are then allowed to omit the derivative $\frac{\mathrm{d} K}{\mathrm{~d} R}$ in (26). Finally, for our purpose (27) must be solved only in the Coulomb zone which extends at threshold to infinity $R=\infty$. Then we are allowed to omit the second derivative of $\kappa$ in (27).

To this end we arrive at the simpler equations

$$
\begin{gathered}
K^{2}=2\left(E-\frac{15}{8 R^{2}}+\frac{C_{0}}{R}+\frac{4 i \kappa}{R^{2}}\right) \\
\frac{8 \kappa^{2}}{R^{2}}=\frac{C_{2}}{2 R}-K \frac{\mathrm{d} \kappa}{\mathrm{d} R}
\end{gathered}
$$

recently presented in [1].

\section{Comparison with Classical Mechanics}

We investigate here the corresponding stationary Hamilton-Jacobi-equation for the same problem, i.e.

$$
\frac{1}{2}\left(\frac{\mathrm{d} S}{\mathrm{~d} R}\right)^{2}+\frac{1}{2 R^{2}}\left(\frac{\mathrm{d} S}{\mathrm{~d} \varphi}\right)^{2}-\frac{C_{0}}{R}-\frac{C_{2}}{2 R}(\varphi-\pi)^{2}=E
$$

and put for the action

$$
S=K R+\kappa(\varphi-\pi)^{2}
$$

As above we allow the wave numbers $K$ and $\kappa$ to depend on the coordinate $R$, i.e. $K=K(R)$ and $\kappa=\kappa(R)$; but we disregard the derivative $\frac{\mathrm{d} K}{\mathrm{~d} R}$ and reject powers higher than $(\varphi-\pi)^{2}$. That leads us to the simple equation

$$
K^{2}=2\left(E+\frac{C_{0}}{R}\right)=0
$$

being different from the above (28). We conclude that both the centrifugal barrier and the imaginary part in (28) are manifestly quantum contributions. In 
contrast to that observation the classical equation for the wavenumber $\kappa$ is the same as above (29).

The derivation of Wannier's threshold ionization law has used the quantum Equations (28) and (29) in the Coulomb zone, i.e. it was sufficient to take into account contributions of the order $1 / R$. Therefore the centrifugal term $\propto 1 / R^{2}$ did not contribute. The Appendix of this paper shows $\kappa \propto \sqrt{R}$; therefore in the Coulomb zone we get $\frac{\kappa}{R^{2}} \propto \frac{1}{R^{\frac{3}{2}}}$, again negligible in the fare Coulomb zone. We conclude that Wannier has been correctly employed classical mechanics for the threshold law. However, the above conclusions are not suffficient for the formation and decay of electron pairs. The quantum terms play then key roles as will be shown in Section 5 .

\section{Formation and Decay of Electron Pairs}

Our development given in Section 3 deserves several comments regarding the pair production of electrons. We start our analysis with Equation (29). Multiplied with the angular term $(\varphi-\pi)^{2}$ we observe that the Coulomb potential term [see (14)] has been replaced according to

$$
\frac{C_{2}}{2 R}(\varphi-\pi)^{2} \Rightarrow \frac{C_{2}}{2 R}(\varphi-\pi)^{2}-K \frac{\mathrm{d} \kappa}{\mathrm{d} R}(\varphi-\pi)^{2}
$$

Equation (33) may be regarded as gap in the potential energy, i.e. the potential ridge has been pressed down. An alternative statement claims that the static potential surface has been deformed. Note that the potential deformation causes a force given by

$$
\boldsymbol{F}=\nabla\left(K \frac{\mathrm{d} \kappa}{\mathrm{d} R}(\kappa-\pi)^{2}\right)
$$

whose radial component reads

$$
F_{R}=\frac{\partial}{\partial R}\left(K \frac{\mathrm{d} \kappa}{\mathrm{d} R}\right)(\varphi-\pi)^{2}
$$

and its angular component is given by

$$
F_{\varphi}=\frac{1}{R} \frac{\partial}{\partial \varphi}\left(K \frac{\mathrm{d} \kappa}{\mathrm{d} R}(\varphi-\pi)^{2}\right)=\frac{2}{R} K \frac{\mathrm{d} \kappa}{\mathrm{d} R}(\varphi-\pi)
$$

That angular force, obviously a momentum dependent fictitious force, is directed across the ridge, and resembles formally to a Lorentz force for a charged particle in a magnetic field given by

$$
\boldsymbol{F}=Q[\boldsymbol{v} \times \boldsymbol{B}]
$$

The similarity becomes immediately visible because (36) may be rewritten in the vectorial form

$$
\boldsymbol{F}_{\varphi}=\frac{2}{R} \frac{\mathrm{d} \kappa}{\mathrm{d} R}(\varphi-\pi)\left[\boldsymbol{K}_{\|} \times \hat{n}\right]
$$

where $\boldsymbol{K}_{\|}$is the component of the radial pair momentum parallel to the ridge 
and $\hat{n}$ is a unit vector orthogonal to the scattering plane. Thus, our radial pair momentum corresponds to the single particle velocity in (37), the normal vector $\hat{n}$ corresponds to the magnetic field $\boldsymbol{B}$, and the factor in front of the vector product in (38) corresponds to the charge $Q$ in (37).

From (38) it is evident that the fictitious force changes sign if the radial momentum $\boldsymbol{K}_{\|}$does. In consequence of that an incoming wave front is directed towards to the top of the ridge, whereas an outgoing wave mode is turned away from the ridge top. This compares favorably with Wannier's [2] finding of two classical trajectories, a converging one and a diverging one. He did not pay further attention to the converging one because he needed only the diverging one for his threshold law. We conclude that the angular force component is the key tool for electronic excitation, in the present study for the pair production.

We are now able to describe the whole process of pair production. When the incoming electron experiences the ridge its curvature is reduced according the Riccati Equation (29). The target electron as well as the projectile electron are pushed towards the top of the ridge, i.e. the electron-electron attraction due to the fictitious force works. Arrived on the top of the ridge the static attraction given by the potential on the ridge $-C_{0} / R$ attracts the pair as a whole towards the nucleus. The pair runs against the centrifugal barrier given by $15 / 8 R^{2}$, see (19) or (26), where reflection occurs, and a collaps of the negative ion has been avoided. The pair is now reflected into the outgoing wave mode. The fictitious force has now changed sign, i.e. the electron-electron interaction has now become repulsive. The pair decays, one electron falls back to a Rydberg orbital whereas the other one escapes. Above threshold both electrons escape. This latter case was treated by Wannier [2]. From highly accurate experimental data we know that the range of validity of the threshold ionization law is a few eV. From that we conclude a lifetime of the pair of the order of $\tau \approx 10^{-15} \mathrm{sec}$.

\section{Outlook}

We have investigated also other atoms with more than two active electrons for equilibrium configurations. To this end we generalize the hyperradius to more than two electrons,

$$
R=\sqrt{\sum r_{i}^{2}}
$$

and study the total electrostatic potential restricted to a hypersphere of radius $R$ for stationary points, i.e.

$$
W=-\sum_{i} \frac{Z}{r_{i}}+\sum_{i<j} \frac{1}{r_{i j}}+\lambda\left(R^{2}-\sum_{i} r_{i}^{2}\right)
$$

must become stationary.

Above the indice $i, j \in[1, \cdots, N] ; N$ being the number of active electrons. We have found the following configurations [7]:

1) $N=2$ corresponds to the pair formation treated above. The electrons sit the ends of a dumbbell. 
2) $N=3$. The electrons sit in the corners of an equilateral triangle; the nucleus is located at the electronic centre of charge [7].

3) $N=4$. The electrons sit at the corners of a tetrahedron; the nucleus is again in the centre of electronic charge [8].

4) $N>4$. No equilibrium configurations seem to be known.

Analogous to the above treatment of two electrons we can find hypersherical coordinates such that one angle describes the fragmentation symbolically referred to as $N \rightarrow(N-1)+1$, and with help of an eventual rotation we can arrive at a cyclic angle in the Laplacian along the hyperspherical equator. We arrive then at a wave equation like (19) except that the constants $C_{0}$ and $C_{2}$ depend on the number of equilibrium electrons.

Applied to the example of three or four active electrons we predict beyond pairs now triples and quadruples. A prototype of a triple configuration may be generated for instance from an alkali earth atom with two valence electrons in a $\left(n s^{2}\right) S$ state. We hit that target with an electron to the threshold of double escape of the target (usually a few eV). In that energy range the projectile electron is attracted by the target electron pair; the three electrons enter into the equilateral triangle configuration. We stress that the electrons do not move on Bohr orbits but the triangle shrinking in size is electrostatically attracted by the nucleus. That motion occurs on the top of the generalized ridge due to the Coulomb potential $-C_{0} / R$. The triple runs now against the generalized centrifugal barrier now given by $6 / R^{2}$, see [1]. The triple returns now under the influence of the repulsive fictitious force, and decays. One electron escapes whereas the other two electrons become trapped into a double Rydberg state of the target atom.

In both cases of complexes, pair or triple, the emitted electron from the complex decay may hit another target atom and creates there a further complex. In this way a complex may travel through a solid without suffering from inelastic collisions; i.e. the complex transports a current without any resistance.

The description of small complexes runs quite generally along the same lines outlined above except that the constants $C_{0}$ and $C_{2}$ have different numerical values for pairs, triples and quadruples. Note however, one difference may not be overlooked: complexes with an odd number of electrons are fermions whereas an even number of electrons allows only for bosons.

\section{Conclusions}

Atomic structure has been traditionally described within the shell model, i.e. each electron moves in a static potential generated by all other electrons moving on circular orbits. The motion takes place in attractive potential valleys. Each electron can be described by a principal quantum number and angular momentum quantum numbers.

That model based on hydrogen-like atoms becomes shaky at multiple excitation and breaks entirely down near thresholds of multiple escape. In that spectral range several electrons move dominantly correlated, and form pairs, triples 
and/or quadruples as we have seen in this paper. The electrons move no longer on approximately circular orbits, but a cloud of highly excited electrons performs a breathing motion caused by diffraction of an electron wave from a potential ridge. The breathing is an entirely novel and unexpected electron motion in an atom. In the spectral range near a threshold of multiple escape a resonance would be located due to the long-range Coulomb interaction in an infinity of one-electron continua. The Fano resonance theory [9] does not apply to that crucial situation. Actually the embedding into a huge number of continua produces the new kind of motion as described here. The main result of the present study has been that quite generally the particle wave diffraction from a potential ridge induces a fictitious force between the constituents of the system under consideration. Moreover, that force may be attractive or repulsive depending on the mode of motion. A few-electron atom is perhaps one of the simplest examples for that situation. We believe that potential surfaces of big molecules show multidimensional ridge structures leading to similar surprising effects.

\section{Acknowledgements}

Financial support during the start of this work by DFG under contract SFB 276 is gratefully acknowledged.

\section{Conflicts of Interest}

The authors declare no conflicts of interest regarding the publication of this paper.

\section{References}

[1] Klar, H. (2018) Physical Review Letters, 120, 053401. https://doi.org/10.1103/PhysRevLett.120.053401

[2] Wannier, G. (1953) Physical Review, 90, 817. https://doi.org/10.1103/PhysRev.90.817

[3] Macek, J.H. (1968) Journal of Physics, B1, 831. https://doi.org/10.1088/0022-3700/1/5/309

[4] Klar, M. and Klar, H. (1980) Journal of Physics, b13, 1057. https://doi.org/10.1088/0022-3700/13/6/014

[5] Sommerfeld, A. (1944) Atombau und Spektrallinien (Vieweg Braunschweig). Vol. 2, $627 \mathrm{ff}$.

[6] Klar, H. (2017) Journal of Modern Physics, 8, 1029. https://doi.org/10.4236/jmp.2017.87065

[7] Schlecht, W. and Klar, H. (1076) Journal of Physics, B9, 1699.

[8] Schlecht, W. (1975) Thesis University Freiburg.

[9] Fano, U. (1967) Physical Review, 124, 1866. https://doi.org/10.1103/PhysRev.124.1866 


\section{Appendix}

We show here how to solve Equations (28) and (29) at threshold $E=0$.

We need a solution only in the Coulomb zone which extends at zero energy up to infinity $R=\infty$. To this end we get from (28)

$$
K=\sqrt{\frac{C_{0}}{R}}
$$

To solve (29) we put $\kappa=\bar{\kappa} \sqrt{R}$ and get after multiplication with $R$ the algebraic equation

$$
8 \bar{\kappa}^{2}=\frac{C_{2}}{2}-\frac{\sqrt{C_{0}}}{2} \bar{\kappa}
$$

with the solutions

$$
\bar{\kappa}_{1,2}=-\frac{\sqrt{C_{0}}}{32} \pm \frac{\sqrt{C_{0}+64 C_{2}}}{32}
$$

This solution justifies that we have disregarded the imaginary portions in (27) and (28) since $\frac{\mathrm{d}^{2} \kappa}{\mathrm{d} R^{2}} \propto R^{-3 / 2}$ and $\frac{\kappa}{R^{2}} \propto R^{-3 / 2}$ are negligible in the fare Coulomb zone. 